\title{
Sivelestat Attenuates Myocardial Reperfusion Injury during Brief Low Flow Postischemic Infusion
}

\author{
Sverre E. Aune, ${ }^{1}$ Steve T. Yeh, ${ }^{1}$ Periannan Kuppusamy, ${ }^{2}$ M. Lakshmi Kuppusamy, ${ }^{2}$ \\ Mahmood Khan, ${ }^{1}$ and Mark G. Angelos ${ }^{1}$ \\ ${ }^{1}$ Department of Emergency Medicine, The Davis Heart Lung Research Institute, The Ohio State University Wexner Medical Center, \\ 750 Prior Hall, 376 W. 10th Avenue, Columbus, OH 43210, USA \\ ${ }^{2}$ The Division of Cardiovascular Medicine, The Davis Heart Lung Research Institute, The Ohio State University Wexner Medical Center, \\ Columbus, $\mathrm{OH} 43210$, USA
}

Correspondence should be addressed to Mark G. Angelos; mark.angelos@osumc.edu

Received 5 March 2013; Accepted 13 April 2013

Academic Editor: Narasimham L. Parinandi

Copyright (C) 2013 Sverre E. Aune et al. This is an open access article distributed under the Creative Commons Attribution License, which permits unrestricted use, distribution, and reproduction in any medium, provided the original work is properly cited.

\begin{abstract}
The neutrophil elastase inhibitor sivelestat (ONO-5046) possesses unknown mechanisms of cardioprotection when infused following global ischemia, even in the absence of neutrophils. Since myocardial ischemia-reperfusion injury is strongly associated with endothelial dysfunction and reactive oxygen species (ROS) generation during reperfusion, we have tested the hypothesis that infusion of sivelestat during postischemic low flow would preserve endothelial and contractile function and reduce infarct size through an ROS-mediated mechanism. Isolated male rat hearts, subjected to global ischemia of 25 minutes, were reperfused with low flow with or without sivelestat followed by a full flow reperfusion. Hearts treated with sivelestat showed a significant improvement of LV contractile function and a reduction in infarct size. Infusion of L-NAME (nonspecific blocker of endothelial nitric oxide synthase (eNOS)) along with sivelestat during reperfusion reversed the preservation of contractile function and infarct size. In vitro EPR spin trapping experiments showed that sivelestat treatment decreased superoxide adduct formation in bovine aortic endothelial cells (BAECs) subjected to hypoxia-reoxygenation. Similarly, dihydroethidine (DHE) staining showed decreased superoxide production in LV sections from sivelestat-treated hearts. Taken together, these results indicate that sivelestat infusion during postischemic low flow reduces infarct size and preserves vasoreactivity in association with decreased ROS formation and the preservation of nitric oxide.
\end{abstract}

\section{Introduction}

Reperfusion of the ischemic myocardium occurs in nearly 2 million people annually in the United States in patients experiencing cardiac arrest, myocardial infarction, or undergoing cardioplegic arrest during cardiac surgery [1]. Various levels of low flow are induced following such ischemic events, most notably in the early moments of reperfusion [2-4]. In the case of cardiac arrest, which affects over 300,000 people annually in the USA, cardiopulmonary resuscitation (CPR) is used by first responders to initiate reperfusion [5]. Currently this low flow reperfusion generated by CPR [6] results in a survivalto-hospital discharge rate for out-of-hospital cardiac arrest patients of $<10 \%$ [7]. Periods of low coronary flow following myocardial ischemia may present early opportunities for pharmacologic treatment of ischemic myocardium prior to full flow reperfusion [8].

There is currently no approved drug on the US market for postischemic reduction of myocardial infarction [9]. The neutrophil elastase inhibitor sivelestat (ONO-5046) [10] has recently been shown to be cardioprotective in several animal studies and in at least one study in humans [11-13]. It has been recently demonstrated that sivelestat preserves LV function when infused after global ischemia in the Langendorf bufferperfused heart, a model of the functioning heart, that is, bereft of neutrophils [14]. The neutrophil-independent cardioprotective mechanisms of sivelestat in the setting of ischemiareperfusion (IR) are unknown.

Reactive oxygen species (ROS) appear to play an important role in reperfusion-induced injury [15], specifically 
superoxide, hydroxyl radical, and hydrogen peroxide [16]. The release of oxygen-derived free radicals concurrent with the reintroduction of oxygen contributes to the pathophysiology of IR injury $[17,18]$. In addition to cellular and mitochondrial injury through oxygen radical-mediated damage, reactive oxygen species signal neutrophil infiltration and are implicated in endothelial dysfunction and smooth muscle injury [19-22]. Specifically, ischemia-reperfusion impairs endothelium-dependent vasorelaxation of coronary microvessels through production of ROS [23]. Excess hydrogen peroxide production during reperfusion damages vascular smooth muscle cells. Conversely reduction of ROS during reperfusion attenuates myocyte and endothelial cell injury following IR [24].

In this study, we tested the hypothesis that infusion of sivelestat during postischemic low flow would reduce infarct size and preserve endothelial and contractile function through an ROS-mediated mechanism. We used the isolated perfused rat heart model of IR and fluorescent microscopy in tissue slices, and electron paramagnetic resonance (EPR) imaging in cultured endothelial cells, to detect the formation of reactive oxygen species. Our results demonstrate a neutrophil-independent mechanism of sivelestat to reduce infarct size and preserve cardiac performance while reducing early ROS formation and preserving endothelial function.

\section{Methods}

2.1. Isolated Buffer-Perfused Rat Hearts. Male SpragueDawley rats (400-500 g) purchased from Harlan Laboratories (Indianapolis, IN, USA) were cared for in accordance with the National Institute of Health $(\mathrm{NIH})$ guidelines and the approval of the Institutional Animal Care and Use Committee. Hearts were isolated and perfused with buffer as previously described [25]. Rats were anesthetized with intraperitoneal sodium pentobarbital $(70 \mathrm{mg} / \mathrm{kg})$ and heparin $(1,000 \mathrm{U} / \mathrm{kg})$. The trachea was cannulated with a 16-gauge angiocath attached to a rodent ventilator (Harvard Apparatus, South Natick, MA, USA). Animals were ventilated with room air at 70 respirations per min with a $2.5 \mathrm{~mL}$ stroke volume. A midsternal thoracotomy was performed to expose the heart and isolate the aorta. The aorta was cannulated in situ and hearts were excised. Retrograde perfusion of the coronary arteries was immediately initiated with warmed $\left(37^{\circ} \mathrm{C}\right)$ modified Krebs-Henseleit buffer $(1.25 \mathrm{mM} \mathrm{CaCl}, 11 \mathrm{mM}$ glucose, $112 \mathrm{mM} \mathrm{NaCl}, 25 \mathrm{mM} \mathrm{NaHCO} 3,5 \mathrm{mM} \mathrm{KCl}, 1.2 \mathrm{mM}$ $\mathrm{MgSO}_{4}, 1 \mathrm{mM} \mathrm{K} \mathrm{PO}_{4}$, and $0.2 \mathrm{mM}$ octanoic acid, bubbled with $95 \% \mathrm{O}_{2} / 5 \% \mathrm{CO}_{2}, \mathrm{pH} 7.4$ ) at a constant perfusion pressure of $75 \mathrm{~mm} \mathrm{Hg}$. A saline-filled latex balloon attached to a pressure transducer was inflated to 5-10 $\mathrm{mm} \mathrm{Hg}$ in the left ventricle (LV) for measurements of LV contractile function. The heart was positioned inside a temperature-controlled glass chamber at $37^{\circ} \mathrm{C}$.

2.2. Experimental Protocol. Global ischemia was induced by completely occluding perfusion flow to the heart. Hearts were randomly assigned to 3 ischemia groups $(n=8$ per group): group 1: $25 \mathrm{~min}$ of global ischemia; group 2: $25 \mathrm{~min}$ of ischemia followed by $3 \mathrm{~min}$ of low flow with perfusion buffer at $4 \mathrm{~mL}$ per min; group 3: $25 \mathrm{~min}$ of ischemia followed by $3 \mathrm{~min}$ of low flow with sivelestat $(100 \mu \mathrm{g}$ per $\mathrm{mL})$ at $4 \mathrm{~mL}$ per min, administered through a side port directly above the aorta by a PHD 2000 Programmable pump (Harvard Apparatus, Holliston, MA, USA). All hearts were subsequently reperfused for $60 \mathrm{~min}$ at $75 \mathrm{~mm} \mathrm{Hg}$.

\subsection{Coronary Flow and Left Ventricular Function. Coronary} flow was continuously monitored by the use of an inline small animal flow meter (Model T206, Transonic Systems Inc., Ithaca, NY, USA) and recorded using an analog-todigital converter box (Digi-Med ASA-400a, Micro-Med, Inc., Louisville, Kentucky, USA). LV pressure was continuously sampled at $30 \mathrm{~Hz}$ and digitally processed with a DigiMed Heart Performance Analyzer (HPA-210a, Micro-Med, Inc.). Heart rate, $\mathrm{dP} / \mathrm{dt}_{\max }, \mathrm{LV}$ systolic pressure, and $\mathrm{LV}$ end diastolic pressure were derived by computer algorithm. Developed pressure was calculated as the difference between systolic and end diastolic pressures. Rate pressure product (RPP) was calculated as the product of heart rate and developed pressure. Hearts that did not achieve an average RPP of at least 20,000 and an average $\mathrm{dP} / \mathrm{dt}_{\max }$ of at least 2,500 in the preischemic baseline stabilization period were excluded from further experimentation. Metrics of LV functional recovery were calculated as percent recovery at the end of experimentation relative to baseline (preischemic) values.

2.4. Acetylcholine-Induced Change in Coronary Flow. Following $60 \mathrm{~min}$ of reperfusion, hearts were infused with $1.0 \mu \mathrm{M}$ acetylcholine chloride for one minute and then switched back to normal perfusion fluid. At this point the recovery in coronary flow was recorded as the maximum coronary flow value within one minute of reflow with normal perfusion fluid. Since perfusion pressure was maintained constant at $75 \mathrm{~mm} \mathrm{Hg}$, increases and decreases in coronary flow were a reflection of endothelial relaxation and constriction, respectively.

2.5. Infarct Size. Following IR, hearts were stained with triphenyltetrazolium chloride (TTC) for the measurement of infarct size, using the method of Ferrera et al. [26]. Under TTC staining, living tissue appears brick red and infarcted tissue appears pale pink or white. Hearts were frozen at $-80^{\circ} \mathrm{C}$, sliced in $2 \mathrm{~mm}$ sections, incubated in $1 \%$ TTC at $37^{\circ} \mathrm{C}$ for $10 \mathrm{~min}$ per side to allow mitochondrial uptake of TTC, and then fixed in formalin prior to photomicrography. Infarct size was measured using MetaVue imaging software (version 6.2r6, Universal Imaging Corp., Downingtown, PA, USA) and reported as a percent of the total left ventricular area.

2.6. Creatine Kinase Release. Coronary effluent was collected from all hearts before ischemia (i.e. in baseline) and at 10, 30 , and 60 minutes of full reperfusion. The effluent was assayed for creatine kinase (CK) content using a standard spectrophotometric assay kit (Stanbio Laboratory, Boerne, $\mathrm{TX})$. Values of CK activity are reported in $\mathrm{U} \cdot \mathrm{L}^{-1}$ [27].

2.7. Measurement of Superoxide in LV Sections by DHE Staining. In another block of experiments using duplicate groups, 
hearts were collected at the end of the $10 \mathrm{~min}$ of reperfusion for measurements of ROS production under fluorescent microscopy, as previously reported [28]. After reperfusion, hearts were embedded in optimal cutting temperature gel, sliced to $5 \mu \mathrm{m}$ thick in a cryotome, and placed on glass slides. After application of $10 \mu \mathrm{M}$ dihydroethidium (DHE), tissue sections were incubated in a light-impermeable chamber at $37^{\circ} \mathrm{C}$ for $30 \mathrm{~min}$. In the presence of superoxide DHE is converted to the red fluorescent hydroxyethidium molecule [29]. Slides were costained with $4^{\prime}$,6-diamidino-2-phenylindole (DAPI) and photographed using a Nikon Eclipse TE 2000U microscope (Tokyo, Japan) equipped with an X-Cite 120 Fluorescence Illumination System (Lumen Dynamics Group Inc., Mississauga, Ontario). Photographs of hydroxyethidium fluorescence were taken under a rhodamine filter (green excitation $550 \mathrm{~nm}$, red emission $573 \mathrm{~nm}$ ). Fluorescent intensity, which positively correlates with superoxide generation in tissue, was quantified using MetaMorph image analysis software (Molecular Devices, Sunnyvale, CA, USA).

2.8. Effect of Nitric Oxide Synthase (NOS) Inhibitor in Isolated Hearts. Additional hearts were isolated to determine if sivelestat exerts cardioprotection in the presence of NOS inhibition. Isolated hearts ( $n=4$ per group) were subjected to $25 \mathrm{~min}$ of ischemia and $3 \mathrm{~min}$ of low flow at $4 \mathrm{~mL}$ per min followed by full reperfusion for 120 minutes. During $3 \mathrm{~min}$ of low flow, hearts were directly infused with either (a) perfusion buffer; (b) sivelestat (100 $\mu$ g per $\mathrm{mL}$ ); (c) the nonspecific NOS blocker N-nitro-L-arginine methyl ester (L-NAME, $100 \mu \mathrm{M}$ ); or (d) L-NAME + sivelestat ( $N=4$ per group). LV function and infarct size were recorded as detailed above.

2.9. Effect of Hypoxia-Reoxygenation in Bovine Aortic Endothelial Cells. To determine the influence of sivelestat on ROS production in endothelial cells, cultured bovine aortic endothelial cells (BAECs) were subjected to hypoxia-reoxygenation in the presence of the spin trap 5,5-dimethyl-1pyrroline-N-oxide (DMPO) [17]. BAECs were cultured in low-glucose DMEM supplemented with 10\% FBS, penicillin/streptomycin, and $0.1 \%$ of Endothelial Cell Growth Supplement (Millipore, Billerica, MA, USA). Cells were cultured in a humidified environment of $5 \% \mathrm{CO}_{2} / 21 \% \mathrm{O}_{2}$. When cells achieved $80-85 \%$ confluence, they were washed, trypsinized, pelleted, and suspended in low-glucose DMEM without phenol red to achieve a cell count of $5 \times 10^{6}$ cells per $\mathrm{mL}$. To achieve hypoxia, cells were placed in a heated chamber at $37.0^{\circ} \mathrm{C}$ and flushed with $100 \%$ nitrogen for $45 \mathrm{~min}$. Immediately following the rapid addition of DMPO (50 mM final concentration), endothelial cells were flushed with oxygen $\left(95 \% \mathrm{O}_{2} / 5 \% \mathrm{CO}_{2}, \mathrm{pH} 7.4\right)$ for one minute at $37.0^{\circ} \mathrm{C}$ in the presence of (a) no drug, (b) sivelestat ( $100 \mu \mathrm{g}$ per $\mathrm{mL}$ final concentration), (c) the xanthine oxidase inhibitor oxypurinol $(500 \mu \mathrm{M})$, or (d) superoxide dismutase (SOD, $1 \mathrm{kU}$ per $\mathrm{mL}$ from bovine erythrocytes (Sigma-Aldrich Co., St. Louis, MO, USA). Cells were then transferred to a quartz flatcell inside an EPR-300 X-band $(9.7 \mathrm{GHz})$ spectrometer. Second peak amplitude of the DMPO-OH spectrum was quantified and taken as an indication of the magnitude of superoxide production.
2.10. Data Analysis. Data was expressed as mean \pm SEM. Statistical significance between groups was calculated by oneway ANOVA followed by the Tukey range test for multiple comparisons. A $P$ value of $<0.05$ was considered statistically significant.

\section{Results}

3.1. Sivelestat Preserved LV Function. Administration of sivelestat during $3 \mathrm{~min}$ of low flow following ischemia improved the recovery of developed pressure, $\mathrm{dP} / \mathrm{dt}_{\max }$, and rate pressure product (RPP) at $60 \mathrm{~min}$ of reperfusion as compared to hearts receiving $25 \mathrm{~min}$ of ischemia or low flow without sivelestat (Figure 1(a)).

3.2. Sivelestat Administration Preserved Vasoreactivity. The vasoconstrictor acetylcholine was infused for one min at the end of $60 \mathrm{~min}$ of reperfusion. Following this, normal buffer was reperfused, and the rebound in coronary flow was taken as a metric of vasoreactivity. Administration of sivelestat significantly improved recovery of coronary flow $(\mathrm{CF})$ following acetylcholine infusion $(8.4 \pm 1.8 \%)$ as compared to hearts receiving $25 \mathrm{~min}$ of ischemia $(3.4 \pm 0.50 \%)$ or low flow without sivelestat $(-0.20 \pm 1.0 \%)$ (Figure $1(\mathrm{~b})$ ).

3.3. Sivelestat Decreased Myocardial Infarct Size. At the end of $60 \mathrm{~min}$ of reperfusion, hearts infused with sivelestat during 3 min of postischemic low flow had significantly lower infarct area $(11 \pm 2.2 \%)$ than hearts given normal perfusion buffer during low flow $(54 \pm 3.4 \%)$ and hearts receiving $25 \mathrm{~min}$ of ischemia with no low flow $(47 \pm 2.1 \%)$. Representative images are shown in Figure 2(a).

3.4. Sivelestat Decreased Myocardial Creatine Kinase Levels. Creatine kinase $(\mathrm{CK})$ release was measured in coronary effluent collected during baseline and at 10, 30, and $60 \mathrm{~min}$ of reperfusion. CK was significantly reduced at $10 \mathrm{~min}$ of reperfusion in hearts treated with sivelestat during 3 min of postischemic low flow as compared to hearts that received $25 \mathrm{~min}$ of ischemia with no low flow (Figure 2(b)).

3.5. Inhibition of eNOS with L-NAME Reversed Cardioprotection from Sivelestat. After 2 hours of full reperfusion the percent infarct size was significantly less in hearts treated with sivelestat compared to L-NAME-treated hearts (sivelestat I.S. $=19 \pm 3.2 \%$ versus L-NAME I.S. $=50 \pm 2.7 \%$ ). This sivelestat-mediated reduction in infarct size was lost in the L-NAME + sivelestat-treated hearts (I.S. $=59 \pm 0.97 \%$ ) (Figure 3(a)). In these same hearts, the percent recovery of rate pressure product (RPP) was significantly greater in sivelestat-treated hearts compared to vehicle-treated hearts (sivelestat RPP $=47 \pm 5.3 \%$ versus vehicle RPP $=28 \pm 4.7 \%$ ) and to L-NAME + sivelestat-treated hearts ( RPP $=26 \pm 2.9 \%$ ) (Figure 3(b)).

3.6. Sivelestat Decreased Superoxide Levels in LV Tissue Sections. The blockade of eNOS by L-NAME reversed the effect of sivelestat by increasing superoxide fluorescence (Figure 4(a)). DHE staining showed that superoxide generation was 


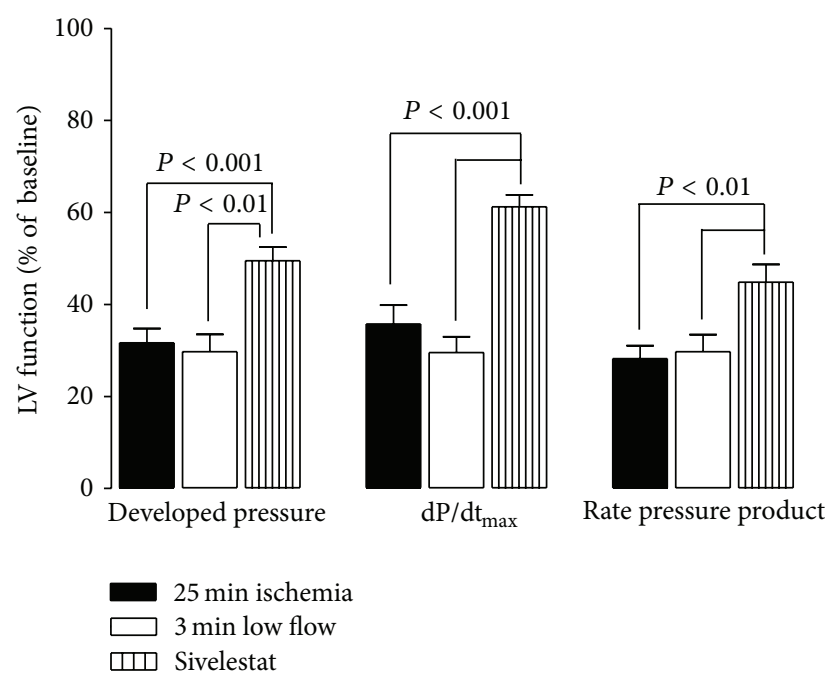

(a)

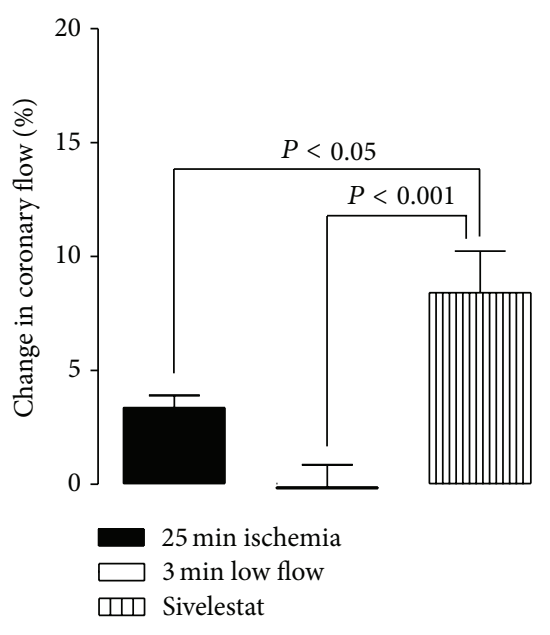

(b)

FIGURE 1: Sivelestat preserves LV and coronary vascular function in ischemia-reperfusion. (a) Effect of sivelestat on LV function and infarct size. Sivelestat significantly improved recovery of developed pressure, $\mathrm{dP} / \mathrm{dt}_{\max }$, and rate pressure product (RPP) as compared to hearts that did not receive low flow ( $25 \mathrm{~min}$ isc) ( $n=8$ per group). (b) Rebound in coronary flow. Sivelestat significantly preserved vasoreactivity at the end of reperfusion as shown through the rebound in coronary flow after one minute of acetylcholine $(1 \mu \mathrm{M})$ infusion $(n=4 /$ group$)$.

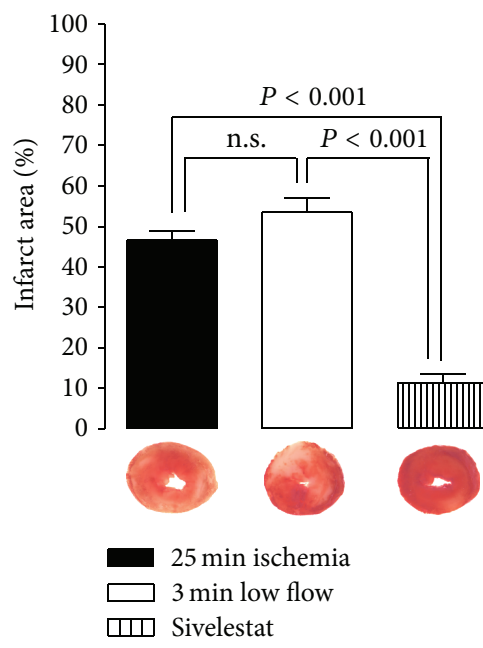

(a)

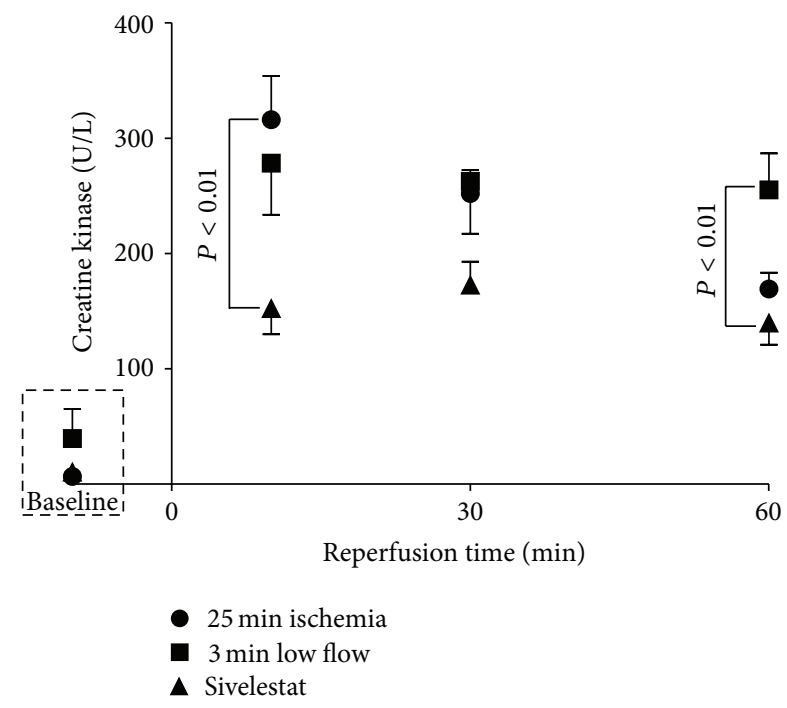

(b)

FIGURE 2: Sivelestat preserves LV tissue in ischemia-reperfusion. (a) Percent infarct area with representative images of left ventricle slices. Sivelestat significantly reduced infarct area as compared to hearts given $25 \mathrm{~min}$ ischemia or ischemia and low flow with vehicle only, as observed through TTC staining. Whiter areas indicate regions of tissue infarction, and pink and red areas indicate functional tissue $(n=4$ per group). n.s.: no significance. (b) Extrusion of intracellular creatine kinase into coronary effluent. Creatine kinase (an intracellular enzyme) release is indicative of cell membrane rupture. Creatine kinase release $(\mathrm{U} / \mathrm{L})$ was significantly reduced by treatment with sivelestat at 10 min of full reperfusion, as compared to hearts that did not receive low flow, and at $60 \mathrm{~min}$ of reperfusion, as compared to vehicle-treated low flow hearts ( $n=8$ /group).

significantly decreased in the sivelestat group (Figure 4(b)). In contrast an increase in superoxide fluorescence was seen in the low flow, L-NAME, and L-NAME + sivelestat treated groups. This data demonstrates the involvement of nitricoxide-mediated cardioprotection by sivelestat.
3.7. Sivelestat Decreased ROS Formation in BAECs. To measure the production of superoxide by hypoxic-reoxygenated bovine aortic endothelial cells (BAECs), DMPO was added at the moment of reoxygenation as a spin trap for superoxide. The EPR-active molecule DMPO-OH is the molecular 


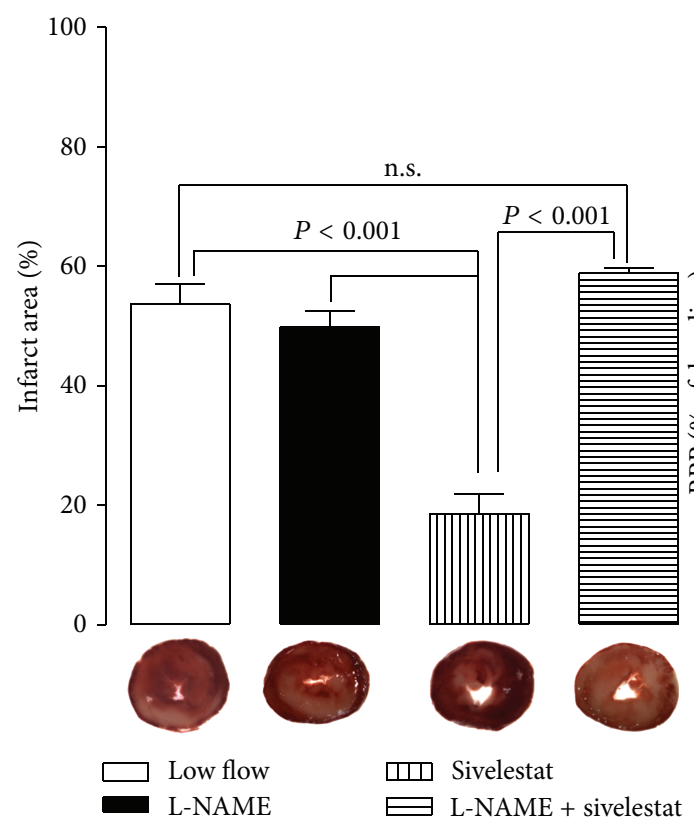

(a)

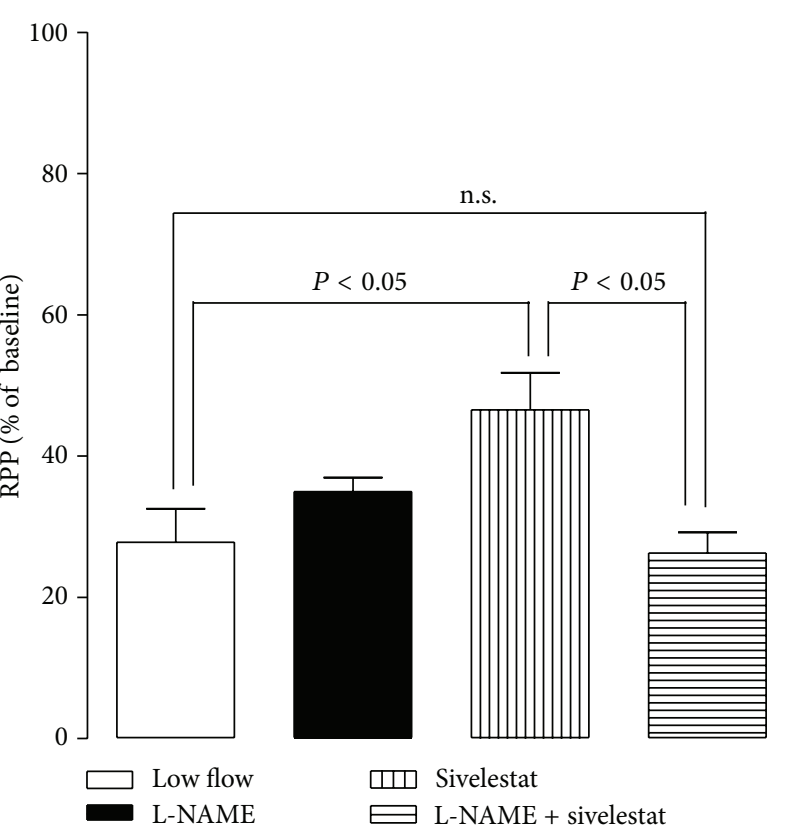

(b)

FIGURE 3: L-NAME reverses the cardioprotective effects of sivelestat in ischemia-reperfusion. (a) Infarct size and (b) recovery of rate pressure product. During low flow, hearts were treated with L-NAME, sivelestat, or both. Though sivelestat preserves RPP and reduces infarct following IR, coinfusion of L-NAME and sivelestat offered no preservation of RPP or infarct reduction as compared to hearts that received vehicle during low flow ( $n=4$ /group). n.s.: nonsignificant.
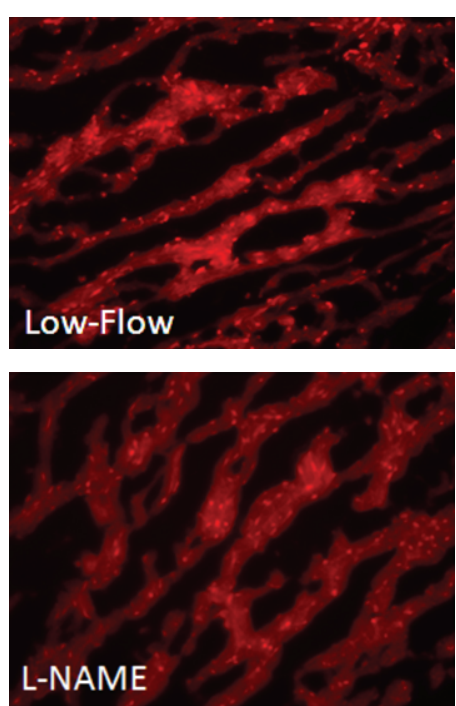
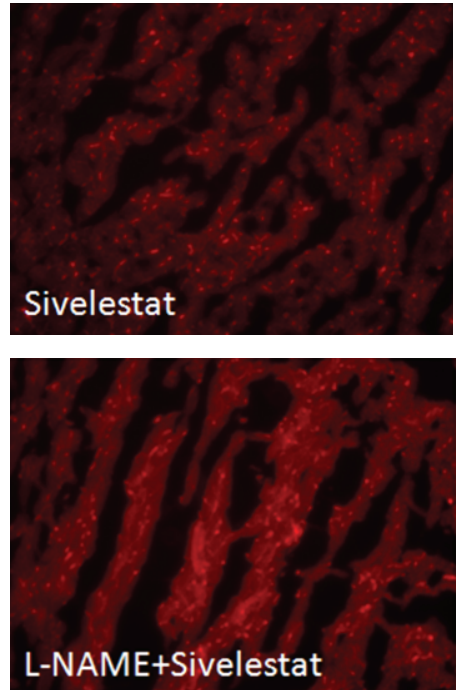

(a)

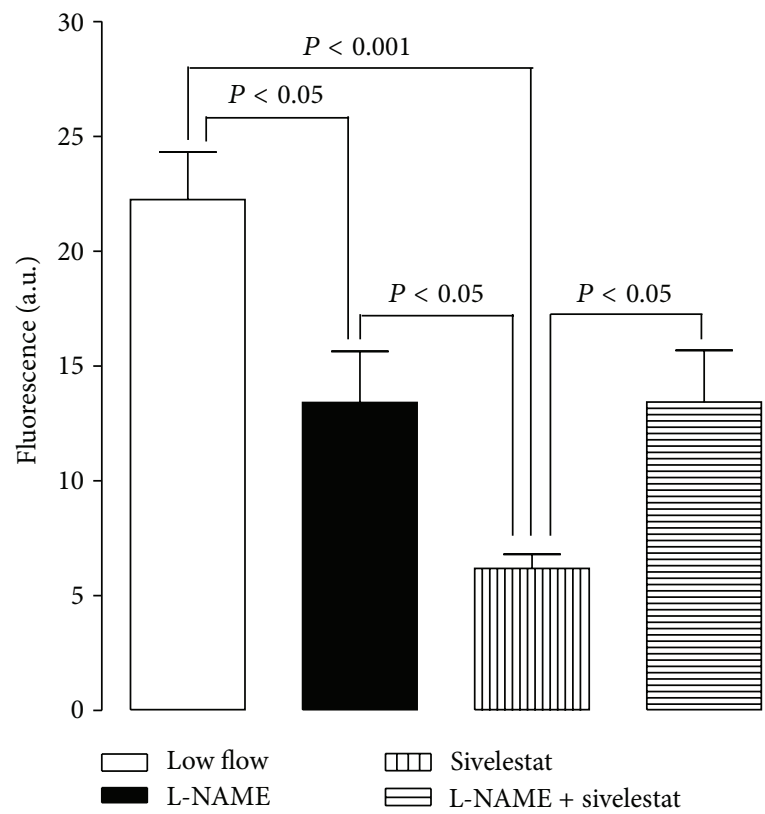

(b)

FIGURE 4: Measurement of ROS in the heart by DHE fluorescence. (a) Representative confocal fluorescence images of cryopreserved LV sections from different groups stained with DHE. (b) Quantification of superoxide generation is indicated by DHE fluorescence, sivelestat decreased tissue ROS during reperfusion, as compared to hearts infused with L-NAME. This decrease was reversed when L-NAME was coinfused with sivelestat ( $n=3-5$ per group). n.s.: nonsignificant. 


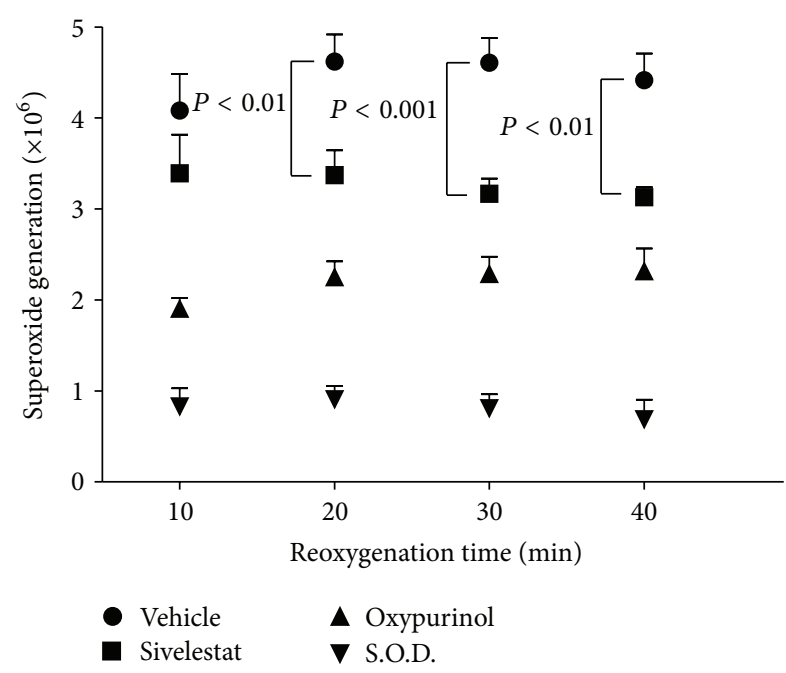

FIGURE 5: EPR spin-trapping of superoxide with DMPO in hypoxicreoxygenated bovine aortic endothelial cells. First integral of DMPO-OH, amplitude of the 2nd spectral peak. DMPO-OH is the stable EPR-active adduct of trapped superoxide. All drugs were applied prior to hypoxia. Sivelestat $(190 \mu \mathrm{M})$ decreased the formation of superoxide (DMPO-OH) in bovine aortic endothelial cells as indicated by spin-trapping with DMPO. Oxypurinol (500 $\mu \mathrm{M})$ was more effective than sivelestat, and S.O.D. $(5 \mathrm{kU} / \mathrm{mL})$ was most effective at decreasing DMPO-OH ( $n=5$ per group).

product of superoxide trapped by DMPO. Sivelestat reduced oxygen radical formation in hypoxic-reoxygenated BAECs exposed to $45 \mathrm{~min}$ of hypoxia followed by reoxygenation. As indicated by the 2nd peak height in the 1st integral of the DMPO-OH EPR spectrum, the spin adduct DMPO-OH was significantly reduced in cells treated with $100 \mu \mathrm{g} / \mathrm{mL}$ sivelestat, as compared to untreated endothelial cells, after $20 \mathrm{~min}, 30 \mathrm{~min}$, and $40 \mathrm{~min}$ of reoxygenation. Oxypurinol significantly reduced $\mathrm{DMPO}-\mathrm{OH}$ formation at every time point as compared to vehicle, but not as effectively as S.O.D. (Figure 5).

\section{Discussion}

In this study, the combination of postischemic low flow with a novel pharmaceutical agent, sivelestat, for 3 minutes at the onset of reperfusion successfully reduced infarct size, improved contractile function, preserved endotheliumdependent vasodilatation, and reduced creatine kinase release, an indicator of tissue destruction at the cellular level [30]. Sivelestat reduced superoxide production in both the ischemia-reperfused explanted LV tissue and the hypoxiareoxygenated cultured endothelial cells. However, the functional cardioprotection and reduction in tissue superoxide generation effected by sivelestat was lost with NOS inhibition. Our study is the first to demonstrate the infarct-reducing property of sivelestat in the ischemia-reperfused heart.

Preservation of LV contractile function is in agreement with Kambe et al., who showed a $35 \%$ recovery of LV developed pressure versus controls (16\%) when sivelestat was infused continuously during the first $10 \mathrm{~min}$ of full reperfusion in isolated rat hearts [14]. Also in agreement with
Kambe et al., sivelestat significantly preserved endotheliumdependent vasorelaxation following IR. That study showed a $15 \%$ increase over controls in the rebound in coronary flow in response to acetylcholine infusion at the end of reperfusion in hearts treated with sivelestat.

The infarct-sparing effects of sivelestat were reversed with simultaneous NOS enzyme inhibition, suggesting that nitric oxide bioavailability is essential during a brief 3-minute period of postischemic low flow. It is likely that sivelestat protects endothelium through dependence on functional NOS enzymes since myocardial protection was abolished by NOS blockade in our study. The idea that preservation of NO bioavailability may lead to myocardial salvage from IR has been widely accepted [31]. Others have shown that sivelestat requires functional NOS enzymes for exertion of protective effects on tissue. Okajima et al. concluded that sivelestat reduced IR-induced liver injury, an effect that was completely inhibited by pretreatment with L-NAME and relied on endothelium-dependent production of NO [32]. Takayama and Uchida showed that sivelestat inhibited substance Pinduced contraction of tracheal ring preparations and that this effect was significantly attenuated by either removal of epithelium or blockade of NOS by L-NAME. However, they concluded that sivelestat exerted vasodilatory effects on guinea pig airways by both NO- and epithelium-dependent and NO- and epithelium independent mechanisms [33].

The retention of endothelium-dependent vasoreactivity with sivelestat may be explained by preservation of sensitivity to nitric oxide in the smooth muscle. However, other studies assert that sivelestat mediates vasorelaxation independent of endothelium. Maeda et al. suggested that this mechanism lies in the vasculature, by demonstrating that sivelestat selectively inhibits calcium sensitization to a receptor agonist in porcine vascular smooth muscle strips with or without endothelium, without affecting calcium-induced contraction [34]. Amemori et al. reiterated this work showing that sivelestat induces endothelium-independent vasorelaxation in precontracted human gastric arteries [35]. It should be noted that sivelestat also protects the heart in situations where neutrophils are present. Akiyama et al. showed attenuation of myocardial stunning in swine with postischemic infusion of sivelestat [11]. Toyama et al. reported an association of sivelestat infusion with improved fractional area of change in the left ventricle of pediatric patients who underwent cardiovascular surgery with cardiopulmonary bypass, demonstrating that sivelestat is protective in at least one setting of ischemiareperfusion in humans [12]. However, our results confirm that sivelestat preserves vasodilatation in the coronary endothelium of hearts subjected to IR in an environment without neutrophils.

The role of eNOS in sivelestat-induced cardioprotection must be further explored. In order to produce nitric oxide from L-arginine, eNOS enzymes require tetrahydrobiopterin $\left(\mathrm{BH}_{4}\right)$ to couple L-arginine oxidation to NADPH consumption and prevent dissociation of the ferrous-dioxygen complex [36]. When $\mathrm{BH}_{4}$ is depleted, as in myocardial ischemia, the association of the ferrous-dioxygen complex can decrease. Once perfusion is resumed, eNOS is not able to couple L-arginine oxidation to NADPH consumption and becomes 


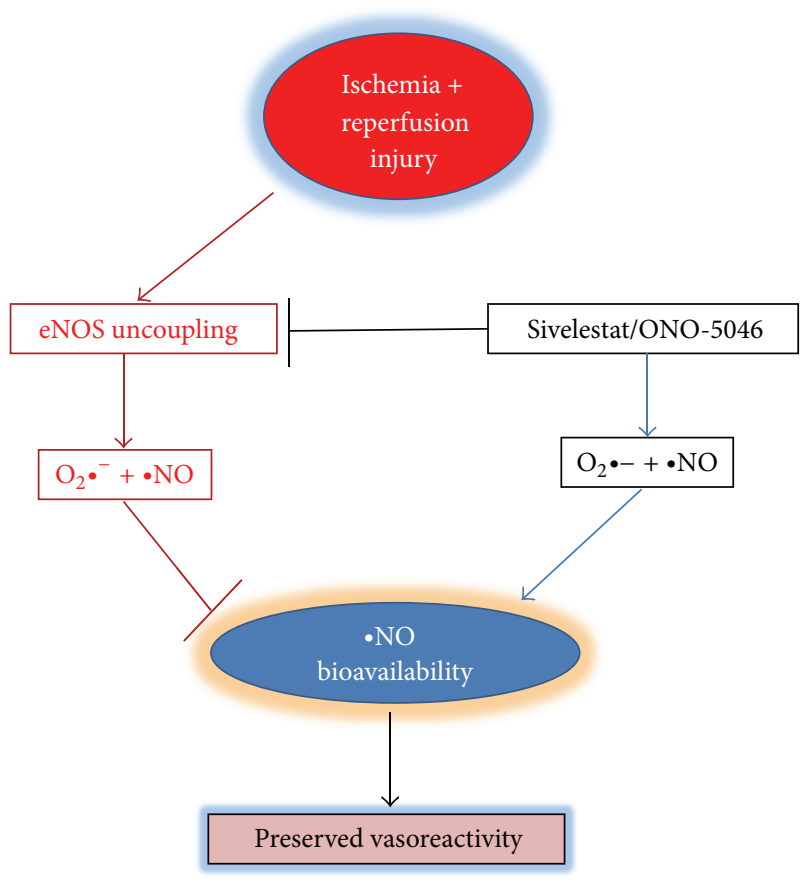

FIGURE 6: Proposed mechanism of sivelestat cardioprotection. During reperfusion, eNOS enzymes are uncoupled from nitric oxide production and produce superoxide in excess. Sivelestat may prevent eNOS uncoupling, thereby reducing superoxide overproduction and preserving nitric oxide production. Nitric oxide bioavailability preserves vascular function in cardiac ischemia-reperfusion.

a true NADPH oxidase, producing superoxide instead of nitric oxide [37]. It is reasonable to hypothesize that sivelestat preserves the association of the ferrous-di-oxygen complex thus preserving nitric oxide bioavailability. In support of this hypothesis we noted an increase in vascular reactivity to acetylcholine and a reduction of superoxide production in aortic endothelial cells (see Figure 6). However, additional studies are needed to elucidate the exact mechanism(s) of protection exerted by sivelestat. For instance, direct measurements of NO production by coronary endothelium are needed to confirm that sivelestat enhances bioavailability of NO.

In summary, our results demonstrate that sivelestat protects the heart against IR injury by scavenging ROS and thereby decreasing the oxidative damage at reperfusion leading to improved LV function and decreased infarct size. The cardioprotective effect of sivelestat may be attributed to an NOS-mediated mechanism. Sivelestat is effective at salvaging tissue when applied in very brief postischemic low flow conditions. This low flow reperfusion differs from the classic mechanical postconditioning, which consists of stuttered start-stop episodes following ischemia [38].

\section{Conclusions}

Overall, these results demonstrate that the period of postischemic low flow can be exploited for significant cardioprotection by pharmaceutical intervention. Due to its availability, promising results in animal studies, and current use in humans, sivelestat is a promising translational pharmaceutical for the investigation of myocardial infarction treatment.

$\begin{array}{ll}\text { Abbreviations } \\ \text { ROS: } & \text { Reactive oxygen species } \\ \text { IR: } & \text { Ischemia-reperfusion } \\ \text { LV: } & \text { Left ventricular } \\ \text { BAEC: } & \text { Bovine aortic endothelial cell } \\ \text { CK: } & \text { Creatine kinase } \\ \text { RPP: } & \text { Rate pressure product } \\ \text { TTC: } & \text { Triphenyltetrazolium chloride } \\ \text { DAPI: } & 4^{\prime}, 6-\text {-Diamidino-2-phenylindole } \\ \text { DHE: } & \text { Dihydroethidium } \\ \text { DMPO: } & \text { 5,5-Dimethyl-1-pyrroline N-oxide } \\ \text { ERP: } & \text { Electron paramagnetic resonance } \\ \text { ONOO- }: & \text { Peroxynitrite } \\ \text { NOS: } & \text { Nitric oxide synthase } \\ \text { L-NAME: } & \text { L-Nitro-arginine methyl ester } \\ \text { O.D.: } & \text { Optical density. }\end{array}$

\section{Acknowledgment}

This study was supported by a Grant from the American Heart Association (60015791) to Mark Angelos.

\section{References}

[1] A. T. Turer and J. A. Hill, "Pathogenesis of myocardial ischemiareperfusion injury and rationale for therapy," American Journal of Cardiology, vol. 106, no. 3, pp. 360-368, 2010.

[2] D. Yannopoulos, S. McKnite, T. P. Aufderheide et al., "Effects of incomplete chest wall decompression during cardiopulmonary resuscitation on coronary and cerebral perfusion pressures in a porcine model of cardiac arrest," Resuscitation, vol. 64, no. 3, pp. 363-372, 2005.

[3] K. Iwakura, H. Ito, S. Takiuchi et al., "Alternation in the coronary blood flow velocity pattern in patients with no reflow and reperfused acute myocardial infarction," Circulation, vol. 94, no. 6, pp. 1269-1275, 1996.

[4] E. R. Rosenkranz and G. D. Buckberg, "Myocardial protection during surgical coronary reperfusion," Journal of the American College of Cardiology, vol. 1, no. 5, pp. 1235-1246, 1983.

[5] M. Montagnana, G. Lippi, M. Franchini, G. Targher, and G. C. Guidi, "Sudden cardiac death: prevalence, pathogenesis, and prevention," Annals of Medicine, vol. 40, no. 5, pp. 360-375, 2008.

[6] D. I. Silver, R. J. Murphy, C. F. Babbs, and L. A. Geddes, "Cardiac output during CPR: a comparison of two methods," Critical Care Medicine, vol. 9, no. 5, pp. 419-420, 1981.

[7] D. F. Gaieski, B. S. Abella, and M. Goyal, "CPR and postarrest care: overview, documentation, and databases," Chest, vol. 141, no. 4, pp. 1082-1089, 2012.

[8] F. Beyersdorf, "The use of controlled reperfusion strategies in cardiac surgery to minimize ischaemia/reperfusion damage," Cardiovascular Research, vol. 83, no. 2, pp. 262-268, 2009.

[9] J. M. Downey and M. V. Cohen, "Why do we still not have cardioprotective drugs?” Circulation Journal, vol. 73, no. 7, pp. 1171-1177, 2009. 
[10] K. Kawabata, M. Suzuki, M. Sugitani, K. Imaki, M. Toda, and T. Miyamoto, "ONO-5046, a novel inhibitor of human neutrophil elastase," Biochemical and Biophysical Research Communications, vol. 177, no. 2, pp. 814-820, 1991.

[11] D. Akiyama, T. Hara, O. Yoshitomi, T. Maekawa, S. Cho, and K. Sumikawa, "Postischemic infusion of sivelestat sodium hydrate, a selective neutrophil elastase inhibitor, protects against myocardial stunning in swine," Journal of Anesthesia, vol. 24, no. 4, pp. 575-581, 2010.

[12] S. Toyama, F. Hatori, A. Shimizu, and T. Takagi, "A neutrophil elastase inhibitor, sivelestat, improved respiratory and cardiac function in pediatric cardiovascular surgery with cardiopulmonary bypass," Journal of Anesthesia, vol. 22, no. 4, pp. 341346, 2008.

[13] M. Ueno, Y. Moriyama, R. Toda et al., "Effect of a neutrophil elastase inhibitor (ONO-5046 Na) on ischemia/reperfusion injury using the left-sided heterotopic canine heart transplantation model," Journal of Heart and Lung Transplantation, vol. 20, no. 8, pp. 889-896, 2001.

[14] M. Kambe, R. Bessho, M. Fujii, M. Ochi, and K. Shimizu, "Sivelestat reduces myocardial ischemia and reperfusion injury in rat hearts even when administered after onset of myocardial ischemia," Interactive Cardiovascular and Thoracic Surgery, vol. 8, no. 6, pp. 629-634, 2009.

[15] B. R. Lucchesi, "Myocardial ischemia, reperfusion and free radical injury," American Journal of Cardiology, vol. 65, no. 19, pp. 14I-23I, 1990.

[16] R. Bolli, "Oxygen-derived free radicals and postischemic myocardial dysfunction ("stunned myocardium")," Journal of the American College of Cardiology, vol. 12, no. 1, pp. 239-249, 1988.

[17] P. Kuppusamy and J. L. Zweier, "Characterization of free radical generation by xanthine oxidase. Evidence for hydroxyl radical generation," Journal of Biological Chemistry, vol. 264, no. 17, pp. 9880-9884, 1989.

[18] V. K. Kutala, M. Khan, M. G. Angelos, and P. Kuppusamy, "Role of oxygen in postischemic myocardial injury," Antioxidants and Redox Signaling, vol. 9, no. 8, pp. 1193-1206, 2007.

[19] K. G. Birukov, "Cyclic stretch, reactive oxygen species, and vascular remodeling," Antioxidants and Redox Signaling, vol. 11, no. 7, pp. 1651-1667, 2009.

[20] X. L. Chen, Q. Zhang, R. Zhao, X. Ding, P. E. Tummala, and R. M. Medford, "Racl and superoxide are required for the expression of cell adhesion molecules induced by tumor necrosis factor- $\alpha$ in endothelial cells," Journal of Pharmacology and Experimental Therapeutics, vol. 305, no. 2, pp. 573-580, 2003.

[21] S. Spiekermann, U. Landmesser, S. Dikalov et al., "Electron spin resonance characterization of vascular xanthine and $\mathrm{NAD}(\mathrm{P}) \mathrm{H}$ oxidase activity in patients with coronary artery disease: relation to endothelium-dependent vasodilation," Circulation, vol. 107, no. 10, pp. 1383-1389, 2003.

[22] J. F. Turrens, "Mitochondrial formation of reactive oxygen species," Journal of Physiology, vol. 552, no. 2, pp. 335-344, 2003.

[23] J. E. Quillen, F. W. Sellke, L. A. Brooks, and D. G. Harrison, "Ischemia-reperfusion impairs endothelium-dependent relaxation of coronary microvessels but does not affect large arteries," Circulation, vol. 82, no. 2, pp. 586-594, 1990.

[24] J. L. Zweier and M. A. H. Talukder, "The role of oxidants and free radicals in reperfusion injury," Cardiovascular Research, vol. 70, no. 2, pp. 181-190, 2006.
[25] J. D. Stoner, T. L. Clanton, S. E. Aune, and M. G. Angelos, " $\mathrm{O}_{2}$ delivery and redox state are determinants of compartmentspecific reactive $\mathrm{O}_{2}$ species in myocardial reperfusion," American Journal of Physiology-Heart and Circulatory Physiology, vol. 292, no. 1, pp. H109-H116, 2007.

[26] R. Ferrera, S. Benhabbouche, J. C. Bopassa, B. Li, and M. Ovize, "One hour reperfusion is enough to assess function and infarct size with ttc staining in langendorff rat model," Cardiovascular Drugs and Therapy, vol. 23, no. 4, pp. 327-331, 2009.

[27] E. C. Dinovo, D. S. Miyada, and R. M. Nakamura, "Evaluation of direct and indirect coupled enzyme assay systems for measurement of creatine kinase activity," Clinical Chemistry, vol. 19, no. 9, pp. 994-997, 1973.

[28] M. Khan, S. Meduru, M. Mostafa, S. Khan, K. Hideg, and P. Kuppusamy, "Trimetazidine, administered at the onset of reperfusion, ameliorates myocardial dysfunction and injury by activation of p38 mitogen-activated protein kinase and akt signaling," Journal of Pharmacology and Experimental Therapeutics, vol. 333, no. 2, pp. 421-429, 2010.

[29] V. P. Bindokas, J. Jordán, C. C. Lee, and R. J. Miller, "Superoxide production in rat hippocampal neurons: selective imaging with hydroethidine," Journal of Neuroscience, vol. 16, no. 4, pp. 13241336, 1996.

[30] F. Kanemitsu and T. Okigaki, "Clinical application of subforms of creatine kinase MM and macro creatine kinases," Journal of Chromatography-Biomedical Applications, vol. 526, no. 2, pp. 423-438, 1990.

[31] Q. D. Wang, J. Pernow, P. O. Sjöquist, and L. Rydén, "Pharmacological possibilities for protection against myocardial reperfusion injury," Cardiovascular Research, vol. 55, no. 1, pp. 25-37, 2002.

[32] K. Okajima, N. Harada, M. Uchiba, and M. Mori, "Neutrophil elastase contributes to the development of ischemia-reperfusion- induced liver injury by decreasing endothelial production of prostacyclin in rats," American Journal of PhysiologyGastrointestinal and Liver Physiology, vol. 287, no. 6, pp. G1116G1123, 2004.

[33] N. Takayama and K. Uchida, "Epithelium-dependent and independent inhibitory effects of sivelestat, a neutrophil elastase inhibitor, on substance P-induced contraction of airway smooth muscle in lipopolysaccharide-treated guinea-pigs," Journal of Smooth Muscle Research, vol. 41, no. 5, pp. 257-270, 2005.

[34] Y. Maeda, S. Mitsumizo, F. Guo et al., "Sivelestat relaxes porcine coronary artery via inhibition of $\mathrm{Ca}^{+}$sensitization induced by a receptor agonist," Journal of Cardiovascular Pharmacology, vol. 51, no. 5, pp. 476-482, 2008.

[35] H. Amemori, Y. Maeda, A. Torikai, and M. Nakashima, "Sivelestat relaxes vascular smooth muscle contraction in human gastric arteries," Journal of Physiology and Biochemistry, vol. 64, no. 4, pp. 589-593, 2011.

[36] J. Vásquez-Vivar, B. Kalyanaraman, P. Martásek et al., "Superoxide generation by endothelial nitric oxide synthase: the influence of cofactors," Proceedings of the National Academy of Sciences of the United States of America, vol. 95, no. 16, pp. 92209225, 1998.

[37] A. L. Moens, R. Kietadisorn, J. Y. Lin, and D. Kass, “Targeting endothelial and myocardial dysfunction with tetrahydrobiopterin," Journal of Molecular and Cellular Cardiology, vol. 51, pp. 559-563, 2011.

[38] H. Kin, Z. Q. Zhao, H. Y. Sun et al., "Postconditioning attenuates myocardial ischemia-reperfusion injury by inhibiting events in 
the early minutes of reperfusion," Cardiovascular Research, vol. 62, no. 1, pp. 74-85, 2004. 


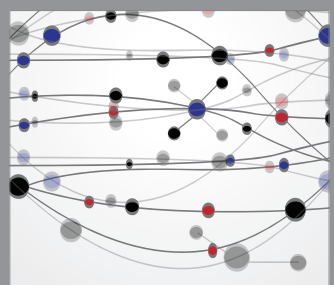

The Scientific World Journal
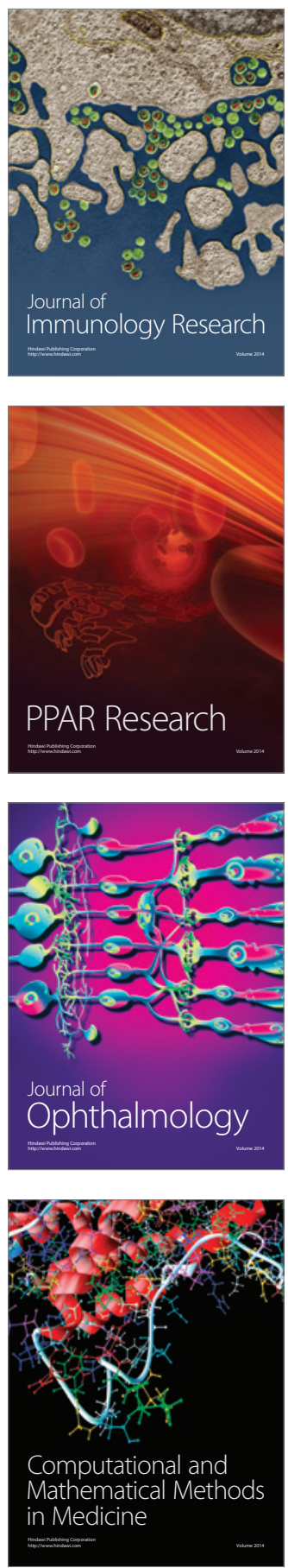

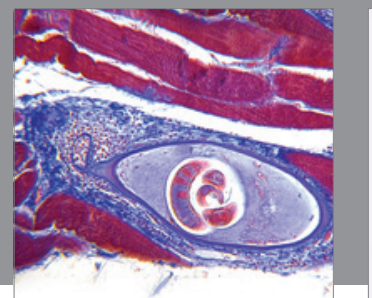

Gastroenterology

Research and Practice
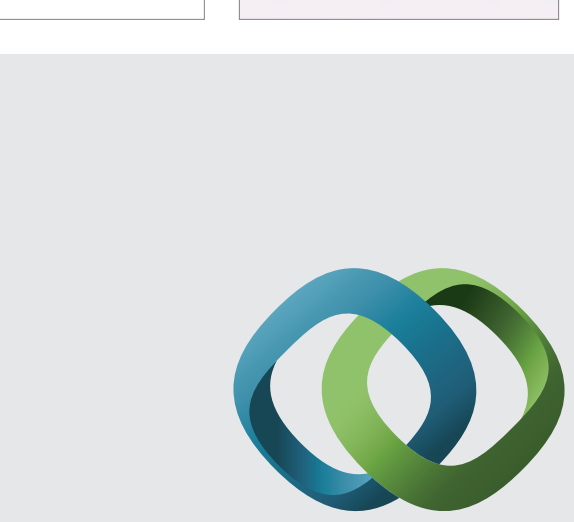

\section{Hindawi}

Submit your manuscripts at

http://www.hindawi.com
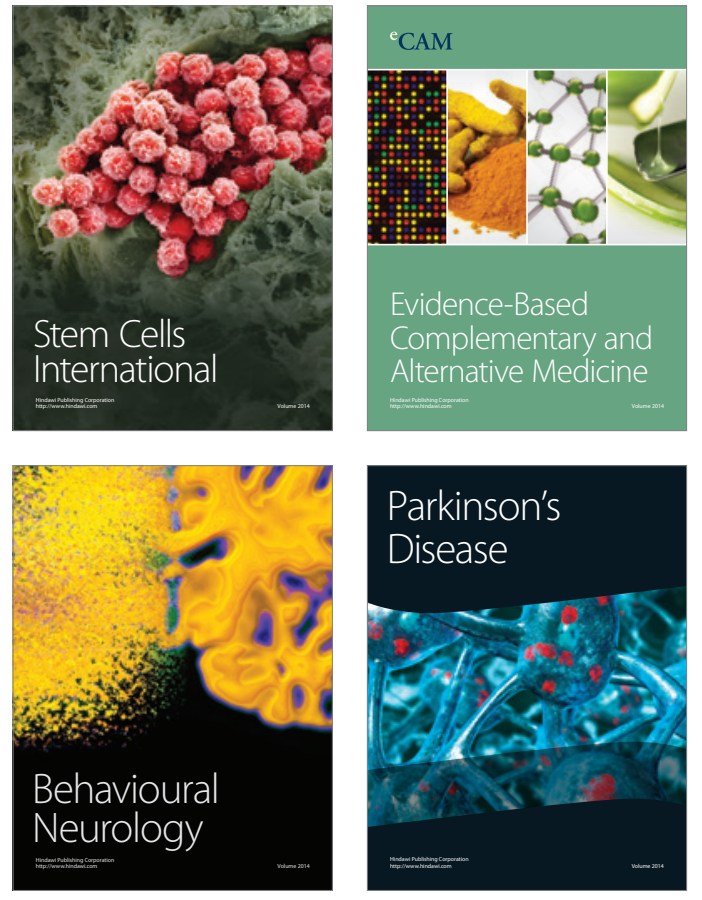
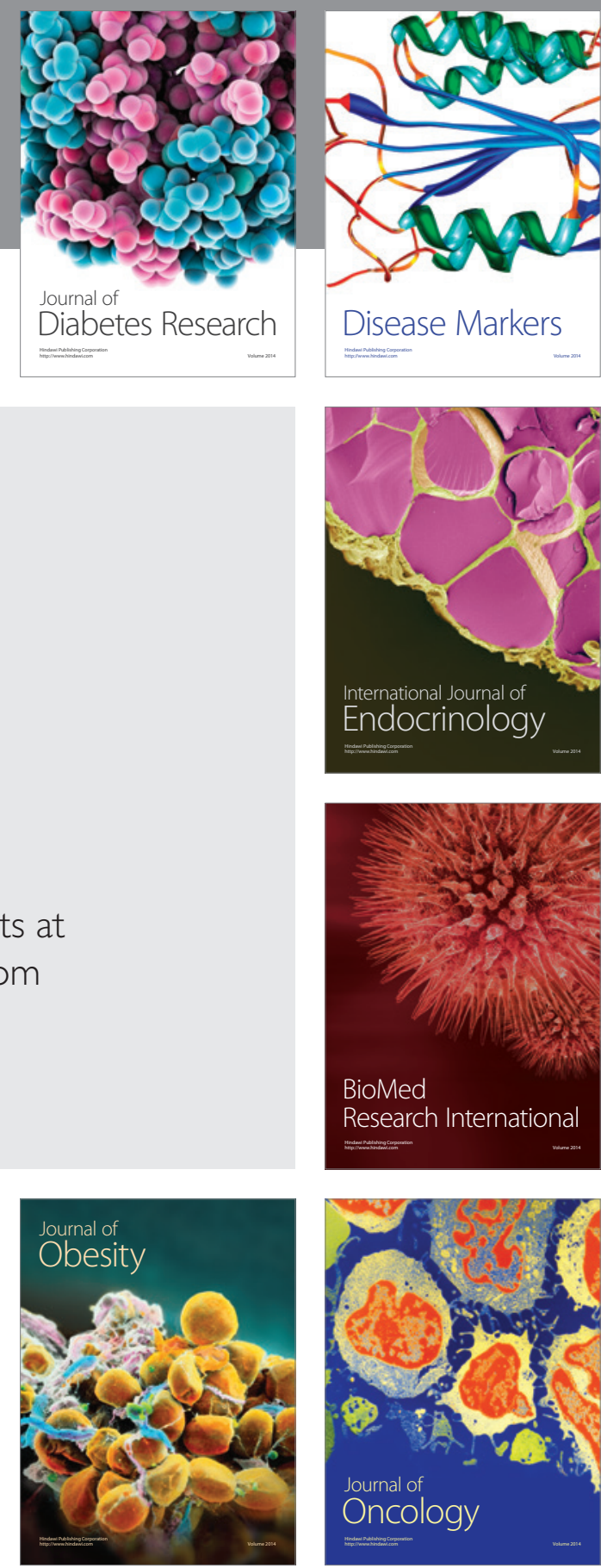

Disease Markers
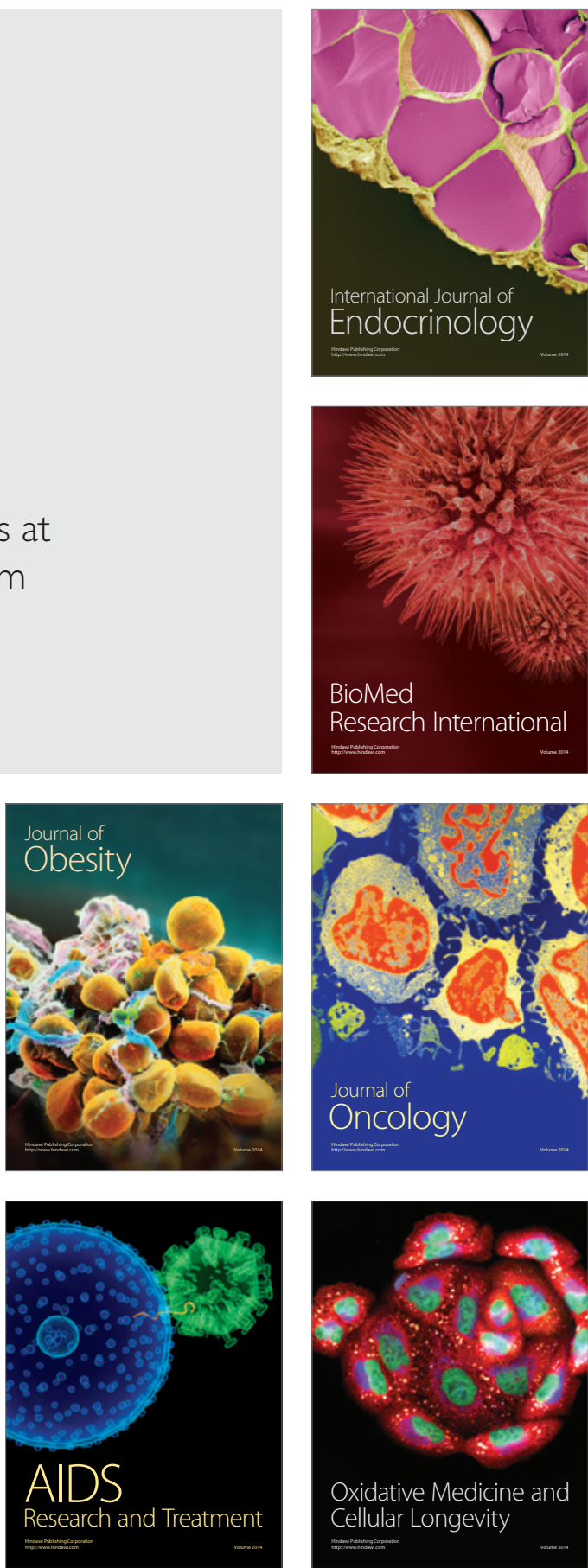